\title{
3D-Modulation and PI State-Feedback Control for Voltage Source Inverters with Split DC-Link
}

\author{
Simon Krüner \\ Graduate School of the \\ Munich School of Engineering (MSE) \\ Technical University of Munich (TUM) \\ Munich, Germany \\ simon.kruener@tum.de
}

\author{
Zhao Song, Christoph M. Hackl \\ Laboratory for Mechatronic and \\ Renewable Energy Systems (LMRES) \\ Munich University of Applied Sciences (MUAS) \\ Munich, Germany \\ \{zhao.song, christoph.hackl\}@hm.edu
}

\begin{abstract}
In this work, a novel three-phase grid inverter topology with split DC-link and LC-filter is discussed. A proportionalintegral (PI) state-feedback current and voltage controller is designed based on linear quadratic regulator (LQR) theory. The midpoint current ripple is suppressed by introducing the filter capacitor voltage as control target. Moreover, a 3-dimensional Space Vector Modulation (3D-SVM) for this topology is implemented, which allows for larger voltage amplitudes compared to conventional Pulse Width Modulation (PWM). Realistic simulation results show that the combination of PI state-feedback controller and 3D-SVM achieves faster and more accurate closedloop dynamics with less oscillations and better decoupling under varying and unbalanced grid conditions.
\end{abstract}

Notation: $\boldsymbol{x}:=\left(x_{1}, \ldots, x_{n}\right)^{\top} \in \mathbb{R}^{n}$ : column vector, $n \in \mathbb{N}$. $\|\boldsymbol{x}\|:=\sqrt{\boldsymbol{x}^{\top} \boldsymbol{x}}$ : Eucilidean norm. $\boldsymbol{A} \in \mathbb{R}^{n \times m}$ : matrix, $n, m \in$ $\mathbb{N}$. $\boldsymbol{O}_{n \times m} \in \mathbb{R}^{n \times m}$ : zero matrix. $\boldsymbol{I}_{n} \in \mathbb{R}^{n \times n}$ : identity matrix.

\section{INTRODUCTION}

Voltage Source Inverters (VSI) play an essential role in renewable energy systems as they connect energy source and grid. VSIs are used in different applications, like in back-to-back converter configurations for controlling electrical machines, e.g. in wind turbine systems [1]. As their applications differ, so do the topologies of VSIs. A lot of research is conducted on multilevel inverters, e.g. to overcome the voltage limitations of the semiconductors [2]. One of the most commonly used multilevel topology is the three-level inverter with neutral point clamping [2], [3]. Here, the DClink capacitor is split into two parts and the midpoint is used as a third output level. In this paper, a three-phase two-level grid-side VSI with six power switches is analysed, which is the standard in low-voltage applications up to $1 \mathrm{kV}$ [4], [5].

Besides the VSI, a grid filter is mandatory to reduce current harmonics and stay within specified limits set by regulations. The state of the art are LCL-filter [5], [6]. For a three-phase inverter, the three capacitors are usually connected in star, where the star-point is either freely floating or connected to the neutral point of the grid. In this paper, a VSI with LC-filter and split DC-link is used. The DC-link midpoint is connected to the star-point of the LC-filter, but not to the grid. To the best of the authors' knowledge, this topology has not been considered in the literature this far. With this connection, an additional degree of freedom in controller design and modulation is provided. As progress in semiconductor technology has enabled higher switching frequencies, here, $70 \mathrm{kHz}$ is chosen, to allow for a wide range where to place the resonant frequency, such that the fundamental components and the controller will not be affected [6].

The grid impedance is not a part of the inverter by itself, but from a control theory perspective, it is also a part of the filter and thus an important parameter. As the grid impedance is becoming more complex due to penetration of decentralized energy resources, its impact on stability of inverter and grid was discussed in a number of papers [7]-[9]. In decentralized systems, the grid impedance can become large, which is usually caused by long cables and low-power transformers [7], [9], [10]. For insufficient controller design, the voltage limits specified by the grid codes could be exceeded, for example by an overshoot or a slow dynamic response of the controller, resulting in a failure of the grid-side VSI [8], [10]. That is why a PI state-feedback controller (PI-SFC) is used here to guarantee a stable current and voltage control under arbitrary operating and grid conditions. The most common controllers are proportional-integral (PI) controllers implemented in the synchronously rotating $(d, q)$-reference frame [9], [10]. Sometimes, proportional-resonant (PR) controllers in the stationary $(\alpha, \beta)$ - or $(a, b, c)$-reference frame are also used [7]. In [11], [12], state-feedback controllers (SFC) are presented for various topologies but without taking the $\gamma$-component into account during modulation and controller design.

Therefore, similar to [13], a 3-dimensional Space Vector Modulation (3D-SVM) is implemented to include also the $\gamma$ voltage as a control signal. The feasible modulation index, which depends on the orientation of the reference voltage vector, can outperform conventional PWM, which is advantageous against abrupt reference changes or disturbances.

The contributions of this paper are: (i) the novel topology for which (ii) a hybrid switching state-space model is established and (iii) the 3D-SVM is extended by dynamic saturation to utilize the full modulation domain (a cube); (iv) an improved anti-windup strategy and (v) the PI-SFC for current and voltage control.

In Section II, the proposed topology is introduced and math- 
ematically modelled. After this, the model is used to design the PI-SFC and the 3D-SVM is presented. In Section IV, the proposed controller is compared to a standard PI controller. It is shown, that the consideration of the $\gamma$-component (additional control objective) is crucial for the proposed topology to work properly.

\section{Modelling}

The considered three-phase grid-side VSI with split DC-link (two DC-link capacitances) and LC-filter is shown in Fig. 1. For this topology, the star-point of the filter capacitor bank (O) is connected to the midpoint of the split DC-link. The neutral (N) point of the grid is not connected to the inverter.

\section{A. Two-Level Voltage Source Inverter}

The inverter applies the voltages $\boldsymbol{u}_{\mathrm{f}}^{a b c}:=\left(u_{\mathrm{f}}^{a}, u_{\mathrm{f}}^{b}, u_{\mathrm{f}}^{c}\right)^{\top}$ to the filter, which can be controlled with the switching state vector $\boldsymbol{s}_{\mathrm{f}}^{a b c}:=\left(s_{\mathrm{f}}^{a}, s_{\mathrm{f}}^{b}, s_{\mathrm{f}}^{c}\right)^{\top}$. For a two-level VSI, there exist eight possible vectors $\boldsymbol{s}_{\mathrm{f}}^{a b c} \in\left\{\boldsymbol{s}_{\mathrm{f}, 1}^{a b c}, \boldsymbol{s}_{\mathrm{f}, 2}^{a b c}, \ldots, \boldsymbol{s}_{\mathrm{f}, 8}^{a b c}\right\}:=$ $\{000,100, \ldots, 111\}$. The three half-bridges of the inverter work in complementary mode, meaning, that if the upper switch is closed $(s=1)$, the lower one must be open $(\bar{s}=0)$, and vice versa. The applied voltages are then

$$
\boldsymbol{u}_{\mathrm{f}}^{a b c}=u_{\mathrm{g}}^{\mathrm{dc}, \mathrm{h}} \boldsymbol{s}_{\mathrm{f}}^{a b c}+u_{\mathrm{g}}^{\mathrm{dc}, 1}\left(\mathbf{1}_{3}-\boldsymbol{s}_{\mathrm{f}}^{a b c}\right),
$$

which depend on $u_{\mathrm{g}}^{\mathrm{dc}, \mathrm{h}}$ and $u_{\mathrm{g}}^{\mathrm{dc}, 1}$. These two voltages may change due to the currents through the split DC-link capacitors. Both capacitors have capacitance $C_{\mathrm{g}}$ and a balancing resistor $R_{\mathrm{b}}$, and the resistors are connected in series to compensate for a voltage drift. With the high- and low-side DC currents $i_{\mathrm{g}}^{\mathrm{dc}, \mathrm{l}}$ and $i_{\mathrm{g}}^{\mathrm{dc}, \mathrm{h}}$ that flow to and from the three half-bridges, the midpoint current $i_{\mathrm{co}}$ can be defined as

$$
i_{\mathrm{co}}=i_{\mathrm{g}}^{\mathrm{dc}, \mathrm{h}}-i_{\mathrm{g}}^{\mathrm{dc}, \mathrm{l}}=\left(\boldsymbol{i}_{\mathrm{f}}^{a b c}\right)^{\top}\left(2 \boldsymbol{s}_{\mathrm{f}}^{a b c}-\mathbf{1}_{3}\right),
$$

where $i_{\mathrm{f}}^{a b c}=\left(i_{\mathrm{f}}^{a}, i_{\mathrm{f}}^{b}, i_{\mathrm{f}}^{c}\right)^{\top}$ are the filter currents. Under the assumption that the DC-link voltage is constant, meaning $\frac{\mathrm{d}}{\mathrm{d} t} u_{\mathrm{g}}^{\mathrm{dc}}=\frac{\mathrm{d}}{\mathrm{d} t} u_{\mathrm{g}}^{\mathrm{dc}, \mathrm{h}}+\frac{\mathrm{d}}{\mathrm{d} t} u_{\mathrm{g}}^{\mathrm{dc}, l} \stackrel{!}{=} 0$, the dynamics of the split capacitor voltages are given by

$$
\left.\begin{array}{c}
\frac{\mathrm{d}}{\mathrm{d} t} u_{\mathrm{g}}^{\mathrm{dc}, \mathrm{h}}=\frac{1}{C_{\mathrm{g}}}\left(\frac{u_{\mathrm{g}}^{\mathrm{dc}}}{2 R_{\mathrm{b}}}-\frac{i_{\mathrm{co}}}{2}-\frac{u_{\mathrm{g}}^{\mathrm{dc}, \mathrm{h}}}{R_{\mathrm{b}}}\right) \\
\frac{\mathrm{d}}{\mathrm{d} t} u_{\mathrm{g}}^{\mathrm{dc}, 1}=\frac{1}{C_{\mathrm{g}}}\left(\frac{u_{\mathrm{g}}^{\mathrm{dc}}}{2 R_{\mathrm{b}}}+\frac{i_{\mathrm{co}}}{2}-\frac{u_{\mathrm{g}}^{\mathrm{dc}, 1}}{R_{\mathrm{b}}}\right) .
\end{array}\right\}
$$

\section{B. LC-Filter}

In this topology, an LC-filter is used. The main purpose of the converter-side inductance $L_{\mathrm{f}}$ is to reduce the current ripple. The capacitor $C_{\mathrm{f}}$ is chosen in order (i) to limit the reactive power, (ii) to keep the power factor high and (iii) to filter the voltages $\boldsymbol{u}_{\mathrm{c}}^{a b c}:=\left(u_{\mathrm{c}}^{a}, u_{\mathrm{c}}^{b}, u_{\mathrm{c}}^{c}\right)^{\top}$ [5]-[7]. In the model, the parasitic resistances $R_{\mathrm{f}}$ and $R_{\mathrm{c}}$ of the filter elements are considered. The resistance $R_{\mathrm{f}}$ is composed of the equivalent series resistance (ESR) of the inductor and the conducting losses of the MOSFETs [14]. Considering both resistors allows for a better controller design and loss calculation. Generally, all resistances act as an additional damping of the system; especially the ESR $R_{\mathrm{c}}$ of the capacitor. An additional resistor is sometimes added in series to the capacitors to further dampen the resonance and simplify a stable operation [7], [15].

\section{Grid Impedance}

The grid inverter is connected at the Point of Common Coupling (PCC) to the grid with grid impedance approximated by $R_{\mathrm{g}}+j \omega L_{\mathrm{g}}$ to model ohmic-inductive characteristics as done in most cases [9]. The grid voltages $\boldsymbol{u}_{\mathrm{g}}^{a b c}:=\left(u_{\mathrm{g}}^{a}, u_{\mathrm{g}}^{b}, u_{\mathrm{g}}^{c}\right)^{\top}$ represent voltage sources, where arbitrarily grid conditions can be defined.

\section{Current Dynamics}

LC-filter and grid-side components are assumed to be symmetric, i.e. all component values are identical in each phase. The current dynamics in the $(a, b, c)$-reference frame are then

$$
\left.\begin{array}{rl}
\frac{\mathrm{d}}{\mathrm{d} t} \boldsymbol{i}_{\mathrm{f}}^{a b c} & =\frac{1}{L_{\mathrm{f}}}\left(\boldsymbol{u}_{\mathrm{f}}^{a b c}-\left(R_{\mathrm{f}}+R_{\mathrm{c}}\right) \boldsymbol{i}_{\mathrm{f}}^{a b c}+R_{\mathrm{c}} \boldsymbol{i}_{\mathrm{g}}^{a b c}-\boldsymbol{u}_{\mathrm{cf}}^{a b c}\right) \\
\frac{\mathrm{d}}{\mathrm{d} t} \boldsymbol{u}_{\mathrm{cf}}^{a b c}=\frac{1}{C_{\mathrm{f}}}\left(\boldsymbol{i}_{\mathrm{f}}^{a b c}-\boldsymbol{i}_{\mathrm{g}}^{a b c}\right) \\
\frac{\mathrm{d}}{\mathrm{d} t} \boldsymbol{i}_{\mathrm{g}}^{a b c}=\frac{1}{L_{\mathrm{g}}}\left(\boldsymbol{u}_{\mathrm{cf}}^{a b c}+R_{\mathrm{c}} \boldsymbol{i}_{\mathrm{f}}^{a b c}-\left(R_{\mathrm{g}}+R_{\mathrm{c}}\right) \boldsymbol{i}_{\mathrm{g}}^{a b c}\right. \\
\left.-\boldsymbol{u}_{\mathrm{g}}^{a b c}-\mathbf{1}_{3} u_{\mathrm{no}}\right)
\end{array}\right\}
$$

where $\boldsymbol{i}_{\mathrm{f}}^{a b c}$ are the filter currents with initial value $\boldsymbol{i}_{\mathrm{f}}^{a b c}(0)=$ $\boldsymbol{i}_{\mathrm{f}, 0}^{a b c}$ and $\boldsymbol{u}_{\mathrm{cf}}^{a b c}=\left(u_{\mathrm{cf}}^{a}, u_{\mathrm{cf}}^{b}, u_{\mathrm{cf}}^{c}\right)^{\top}$ denotes the voltages across filter capacitances with $\boldsymbol{u}_{\mathrm{cf}}^{a b c}(0)=\boldsymbol{u}_{\mathrm{cf}, 0}^{a b c}$. The grid-side currents are $\boldsymbol{i}_{\mathrm{g}}^{a b c}=\left(i_{\mathrm{g}}^{a}, i_{\mathrm{g}}^{b}, i_{\mathrm{g}}^{c}\right)^{\top}$ with $\boldsymbol{i}_{\mathrm{g}}^{a b c}(0)=\boldsymbol{i}_{\mathrm{g}, 0}^{a b c}$ and the voltage $u_{\mathrm{no}}$ describes the potential difference between the star-point of capacitor bank and grid. Since $i_{\mathrm{g}}^{a}+i_{\mathrm{g}}^{b}+i_{\mathrm{g}}^{c}=0$ holds for the star-connected grid side, $u_{\text {no }}$ is given by

$$
u_{\mathrm{no}}=\frac{1}{3}\left(\mathbf{1}_{3}\right)^{\top}\left(\boldsymbol{u}_{\mathrm{cf}}^{a b c}+R_{\mathrm{c}} \boldsymbol{i}_{\mathrm{f}}^{a b c}-\boldsymbol{u}_{\mathrm{g}}^{a b c}\right) .
$$

With the grid voltage angle $\phi_{\mathrm{g}}$ estimated by a phase-locked loop or a frequency-locked loop [16], the Clarke-Park transformation can be applied to yield a representation in the synchronously rotating $(d, q, \gamma)$-reference frame for controller design, with (5) inserted, (4) becomes

$$
\left.\begin{array}{r}
\frac{\mathrm{d}}{\mathrm{d} t} \boldsymbol{i}_{\mathrm{f}}^{d q \gamma}=\frac{1}{L_{\mathrm{f}}}\left(\boldsymbol{u}_{\mathrm{f}}^{d q \gamma}-\left(R_{\mathrm{f}}+R_{\mathrm{c}}+\omega_{\mathrm{g}} L_{\mathrm{f}} \boldsymbol{J}_{0}\right) \boldsymbol{i}_{\mathrm{f}}^{d q \gamma}\right. \\
\left.+R_{\mathrm{c}} \boldsymbol{i}_{\mathrm{g}}^{d q \gamma}-\boldsymbol{u}_{\mathrm{cf}}^{d q \gamma}\right) \\
\frac{\mathrm{d}}{\mathrm{d} t} \boldsymbol{u}_{\mathrm{cf}}^{d q \gamma}=\frac{1}{C_{\mathrm{f}}}\left(\boldsymbol{i}_{\mathrm{f}}^{d q \gamma}-\omega_{\mathrm{g}} C_{\mathrm{f}} \boldsymbol{J}_{0} \boldsymbol{u}_{\mathrm{cf}}^{d q \gamma}-\boldsymbol{i}_{\mathrm{g}}^{d q \gamma}\right) \\
\frac{\mathrm{d}}{\mathrm{d} t} \boldsymbol{i}_{\mathrm{g}}^{d q \gamma}=\frac{1}{L_{\mathrm{g}}}\left[\boldsymbol{T}_{\alpha \beta}\left(\boldsymbol{u}_{\mathrm{cf}}^{d q \gamma}+R_{\mathrm{c}}\left(\boldsymbol{i}_{\mathrm{f}}^{d q \gamma}-\boldsymbol{i}_{\mathrm{g}}^{d q \gamma}\right)-\boldsymbol{u}_{\mathrm{g}}^{d q \gamma}\right)\right. \\
\left.-\left(R_{\mathrm{g}}+\omega_{\mathrm{g}} L_{\mathrm{g}} \boldsymbol{J}_{0}\right) \boldsymbol{i}_{\mathrm{g}}^{d q \gamma}\right]
\end{array}\right\}
$$

where, $\omega_{\mathrm{g}}$ denotes the angular grid frequency and the matrices

$$
\boldsymbol{J}_{0}:=\left[\begin{array}{ccc}
0 & -1 & 0 \\
1 & 0 & 0 \\
0 & 0 & 0
\end{array}\right] \text { and } \boldsymbol{T}_{\alpha \beta}:=\left[\begin{array}{ccc}
1 & 0 & 0 \\
0 & 1 & 0 \\
0 & 0 & 0
\end{array}\right],
$$

reflect the coupling between the axes and that $i_{\mathrm{g}}^{\gamma}=0$. The applied Clarke and Park transformation matrices are

$$
\boldsymbol{T}_{\mathrm{c}}:=\kappa\left[\begin{array}{ccc}
1 & -\frac{1}{2} & -\frac{1}{2} \\
0 & \frac{\sqrt{3}}{2} & -\frac{\sqrt{3}}{2} \\
\frac{1}{\sqrt{2}} & \frac{1}{\sqrt{2}} & \frac{1}{\sqrt{2}}
\end{array}\right] \text { and } \boldsymbol{T}_{\mathrm{p}}\left(\phi_{\mathrm{g}}\right):=\left[\begin{array}{ccc}
\cos \left(\phi_{\mathrm{g}}\right) & -\sin \left(\phi_{\mathrm{g}}\right) & 0 \\
\sin \left(\phi_{\mathrm{g}}\right) & \cos \left(\phi_{\mathrm{g}}\right) & 0 \\
0 & 0 & 1
\end{array}\right]
$$




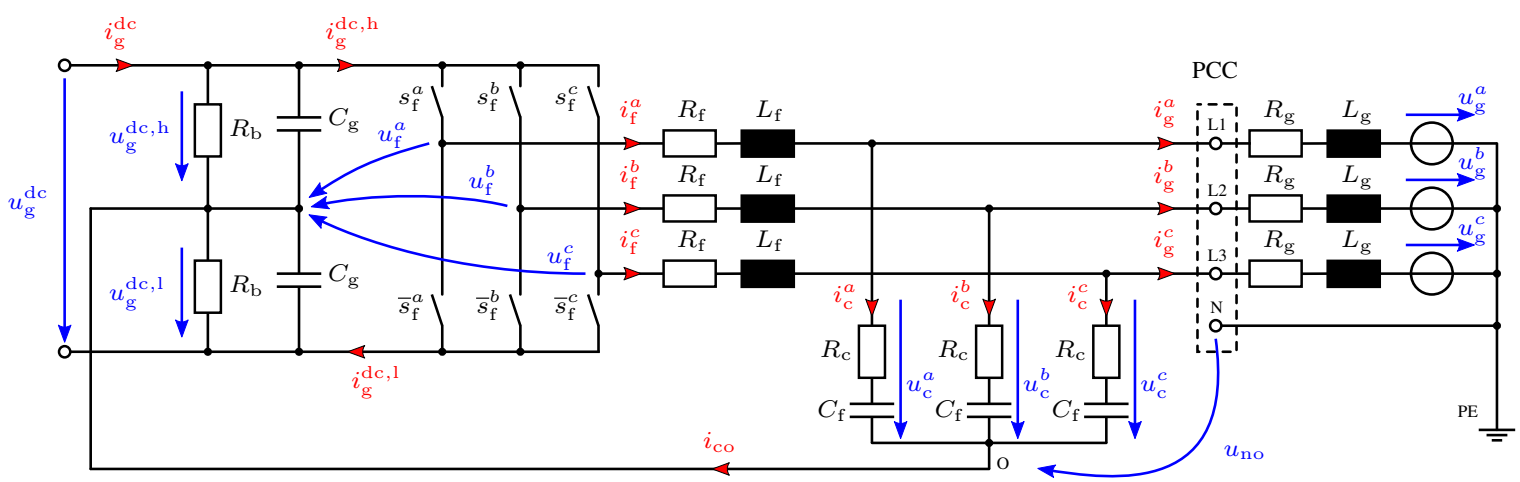

Fig. 1: Electrical circuit of the grid inverter with split capacitance and LC-filter.

respectively, with coefficient $\kappa=\frac{2}{3}$ gives an amplitude-correct transformation. Now, the state space representation is

$$
\begin{aligned}
& \frac{\mathrm{d}}{\mathrm{d} t} \boldsymbol{x}=\underbrace{\left[\begin{array}{cc}
-\frac{R_{\mathrm{f}}+R_{\mathrm{c}}}{L_{\mathrm{f}}} \boldsymbol{I}_{3}-\omega_{\mathrm{g}} \boldsymbol{J}_{0} & -\frac{1}{L_{\mathrm{f}}} \boldsymbol{I}_{3} \\
\frac{1}{C_{\mathrm{f}}} \boldsymbol{I}_{3} & -\omega_{\mathrm{g}} \boldsymbol{J}_{0}
\end{array}\right]}_{:=\boldsymbol{A}} \boldsymbol{x}+\underbrace{\left[\begin{array}{c}
\frac{1}{L_{\mathrm{f}}} \boldsymbol{I}_{3} \\
\boldsymbol{O}_{3 \times 3}
\end{array}\right]}_{:=\boldsymbol{B}} \boldsymbol{u} \\
& +\underbrace{\left[\begin{array}{c}
\frac{R_{\mathrm{c}}}{L_{\mathrm{f}}} \boldsymbol{I}_{3} \\
-\frac{1}{C_{\mathrm{f}}} \boldsymbol{I}_{3}
\end{array}\right]}_{:=\boldsymbol{B}_{\mathrm{d}}} \boldsymbol{u}_{\mathrm{d}}, \\
& \boldsymbol{y}=\underbrace{\left[\begin{array}{llllll}
1 & 0 & 0 & 0 & 0 & 0 \\
0 & 1 & 0 & 0 & 0 & 0 \\
0 & 0 & 0 & 0 & 0 & 1
\end{array}\right]}_{:=C} \boldsymbol{x}
\end{aligned}
$$

having state vector $\boldsymbol{x}:=\left(\left(\boldsymbol{i}_{\mathrm{f}}^{d q \gamma}\right)^{\top},\left(\boldsymbol{u}_{\mathrm{cf}}^{d q \gamma}\right)^{\top}\right)^{\top} \in \mathbb{R}^{6}$, input $\boldsymbol{u}:=\boldsymbol{u}_{\mathrm{f}}^{d q \gamma} \in \mathbb{R}^{3}$, disturbance $\boldsymbol{u}_{\mathrm{d}}:=\boldsymbol{i}_{\mathrm{g}}^{d q \gamma} \in \mathbb{R}^{3}$ and output $\boldsymbol{y}:=\left(i_{\mathrm{f}}^{d}, i_{\mathrm{f}}^{q}, u_{\mathrm{cf}}^{\gamma}\right)^{\top} \in \mathbb{R}^{3}$ with the controlled states. In general and addition, an observer can also be implemented (see [12]).

\section{CONTROL}

Based on (9), a state-feedback controller is designed.

\section{A. Choice of controlled variables}

Here, the converter-side currents should be controlled and thus are chosen as controlled variables. In the proposed topology, $i_{\mathrm{f}}^{\gamma}$ has to flow through the capacitors to the midpoint of split DC-link, which gives the relation

$$
\frac{i_{\mathrm{f}}^{\gamma}(s)}{u_{\mathrm{f}}^{\gamma}(s)}=\frac{s C_{\mathrm{f}}}{1+s\left(R_{\mathrm{f}}+R_{\mathrm{c}}\right) C_{\mathrm{f}}+s^{2} L_{\mathrm{f}} C_{\mathrm{f}}} .
$$

This transfer function describes a resonant RLC circuit, where current resonances can occur due to the non-zero voltage of $u_{\mathrm{f}}^{\gamma}$ during the modulation, even its reference is set to zero. This makes the resonance difficult to be compensated for directly by $u_{\mathrm{f}}^{\gamma}$. But, in view of with the relation

$$
i_{\mathrm{f}}^{\gamma}=i_{\mathrm{c}}^{\gamma}=C_{\mathrm{f}} \frac{\mathrm{d}}{\mathrm{d} t} u_{\mathrm{cf}}^{\gamma},
$$

the resonance shall be suppressed if $u_{\mathrm{cf}}^{\gamma}$ is controlled to zero. Therefore, $u_{\mathrm{cf}}^{\gamma}$ is chosen as controlled variable instead of $i_{\mathrm{f}}^{\gamma}$. Another advantage of controlling $u_{\mathrm{c}}^{\gamma}$ to zero is related to the voltage shift between the split DC-link capacitors. Neglecting the current flow through the balancing resistors $R_{\mathrm{b}}$ due to their high resistance, the voltage difference $\Delta u_{\mathrm{g}}^{\mathrm{dc}}$ is defined as

$$
\Delta u_{\mathrm{g}}^{\mathrm{dc}}=u_{\mathrm{g}}^{\mathrm{dc}, \mathrm{h}}-u_{\mathrm{g}}^{\mathrm{dc}, 1}=-\frac{3 C_{\mathrm{f}}}{\sqrt{2} C_{\mathrm{g}}} u_{\mathrm{c}}^{\gamma},
$$

where (2) and $i_{\mathrm{co}}=\left(\mathbf{1}_{3}\right)^{\top} \boldsymbol{T}_{\mathrm{c}}^{-1} \boldsymbol{i}_{\mathrm{c}}^{a b c}=\frac{3}{\sqrt{2}} i_{\mathrm{c}}^{\gamma}$ were used. $\Delta u_{\mathrm{g}}^{\mathrm{dc}}$ can therefore also be eliminated if $u_{\text {cf }}^{\gamma}$ is controlled to zero.

\section{B. 3-Dimensional Space Vector Modulation (3D-SVM)}

By applying the Clarke transformation as in (8) with $\kappa=\frac{2}{3}$ to (1), the eight possible output vectors $\boldsymbol{v}^{\alpha \beta \gamma} \in$ $\left\{\boldsymbol{v}_{1}^{\alpha \beta \gamma}, \boldsymbol{v}_{2}^{\alpha \beta \gamma}, \ldots, \boldsymbol{v}_{8}^{\alpha \beta \gamma}\right\}$ of VSI for all switching states can be derived. The two zero vectors $\boldsymbol{v}_{1}^{\alpha \beta \gamma}$ and $\boldsymbol{v}_{8}^{\alpha \beta \gamma}$ contribute only to the $\gamma$-component, while the other six active vectors possess now a $\gamma$-component besides their $\alpha$ - and $\beta$-components. The feasible domain of the 3D-SVM extends from the known hexagon formed by six active vectors to a cube formed by all the eight voltage vectors as shown in Fig. 2a. Each space vector starts from the center point and reaches out along one vertex of the cube with same amplitude $\frac{\sqrt{2}}{2} u_{\mathrm{g}}^{\mathrm{dc}}$. Each two adjacent active vectors together with the zero vectors form a tetrahedron, which corresponds to the sectors projected onto the $(\alpha, \beta)$-plane (see Fig. 2b). The tetrahedron can be equally divided further into two sub-tetrahedrons by a separation plane extending from the center point to the side of the cube. For example, in Fig. 2, the sub-tetrahedron formed by $\boldsymbol{v}_{2}^{\alpha \beta \gamma}, \boldsymbol{v}_{3}^{\alpha \beta \gamma}$ and $\boldsymbol{v}_{8}^{\alpha \beta \gamma}$ is shown, whose correspondent larger tetrahedron is formed by $\boldsymbol{v}_{1}^{\alpha \beta \gamma}, \boldsymbol{v}_{2}^{\alpha \beta \gamma}, \boldsymbol{v}_{3}^{\alpha \beta \gamma}$ and $\boldsymbol{v}_{8}^{\alpha \beta \gamma}$. The respective vectors can be used to synthesize the reference vectors located in it. In order to obtain the switching pattern or duty cycle, the required tetrahedron and sub-tetrahedron must be identified by following two steps:

(i) Find the sector by projecting the reference vector to the $(\alpha, \beta)$-plane, like in Fig. $2 \mathrm{~b}$.

(ii) Identify the sub-tetrahedron considering now the $\gamma$ component. By defining the normal vector of the separation surface $\boldsymbol{v}_{\mathrm{n}}:=\left\{\alpha_{\mathrm{n}}, \beta_{\mathrm{n}}, \gamma_{\mathrm{n}}\right\}, \gamma_{\mathrm{n}} \geq 0$, the reference vector is localized in the upper sub-tetrahedron formed by $\boldsymbol{v}_{8}^{\alpha \beta \gamma}$ if $\boldsymbol{v}_{\mathrm{n}}^{\top} \boldsymbol{u}_{\mathrm{f}, \mathrm{ref}}^{\alpha \beta \gamma}>0$, otherwise by $\boldsymbol{v}_{1}^{\alpha \beta \gamma}$.

For a given reference voltage vector $\boldsymbol{u}_{\mathrm{f}, \mathrm{ref}}^{\alpha \beta \gamma}$, the 3D-SVM distributes the switching states during a switching period to the two identified active vectors $\boldsymbol{v}_{\mathrm{a}, 1}^{\alpha \beta \gamma}, \boldsymbol{v}_{\mathrm{a}, 2}^{\alpha \beta \gamma}$ and the two zero vectors for synthesization, such that the time distribution

$$
\left(\begin{array}{c}
T_{1} \\
T_{\mathrm{a}, 1} \\
T_{\mathrm{a}, 2} \\
T_{8}
\end{array}\right)=\left[\begin{array}{cccc}
\boldsymbol{v}_{1}^{\alpha \beta \gamma} & \boldsymbol{v}_{\mathrm{a}, 1}^{\alpha \beta \gamma} & \boldsymbol{v}_{\mathrm{a}, 2}^{\alpha \beta \gamma} & \boldsymbol{v}_{8}^{\alpha \beta \gamma} \\
1 & 1 & 1 & 1
\end{array}\right]^{-1}\left(\begin{array}{c}
T_{\mathrm{s}} \boldsymbol{u}_{\mathrm{f}, \mathrm{ref}}^{\alpha \beta \gamma} \\
T_{\mathrm{s}}
\end{array}\right)
$$

can be obtained [13]. In order to find a feasible solution, and to fully utilize the feasible modulation space, a proper 


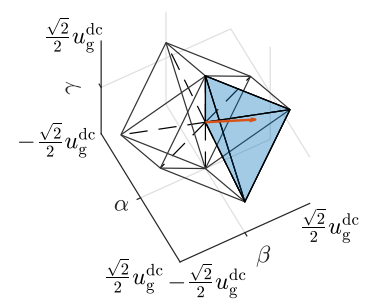

(a)

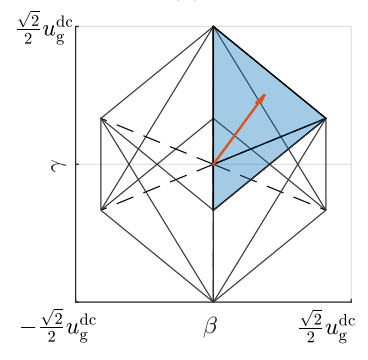

(c)

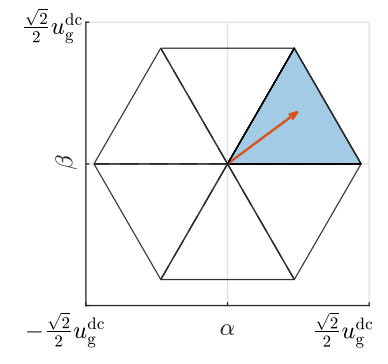

(b)

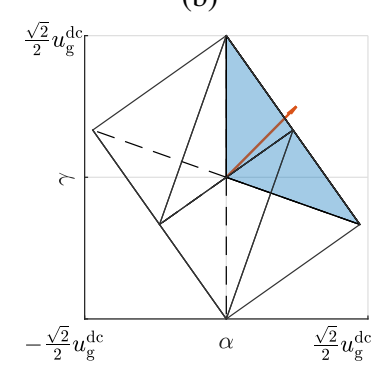

(d)
Fig. 2: 3D-SVM domain with exemplary reference vector (red). Feasible modulation cube (a) formed by eight space vectors (dashed). Projection onto $(\alpha, \beta)$-plane (b), $(\beta, \gamma)$-plane (c) and $(\alpha, \gamma)$-plane (d).

saturation of the reference vector must be applied which limits the magnitude of the vector to the cube's surface while keeping its original orientation [17]. For the exemplary reference, it can be seen that its projection onto the $(\alpha, \gamma)$-plane in Fig. $2 d$ exceeds the modulation domain. The saturation is realized in the identified sub-tetrahedron as follows:

(i) Project the center point $o$ of the cube to the limit plane of the identified sub-tetrahedron orthogonally as new center point $o^{\prime}$. The distance between them is $\left\|\boldsymbol{v}_{o o^{\prime}}^{\alpha \beta \gamma}\right\|=\frac{1}{\sqrt{6}} u_{\mathrm{g}}^{\mathrm{dc}}$.

(ii) Calculate the angle $\theta_{\text {ref }}$ between $\boldsymbol{u}_{\mathrm{f}, \mathrm{ref}}^{\alpha \beta \gamma}$ and $\boldsymbol{v}_{o o^{\prime}}^{\alpha \beta \gamma}$, the maximal possible amplitude of the same reference direction is $\left\|\boldsymbol{u}_{\mathrm{f}}^{\alpha \beta \gamma}\right\|_{\max }:=\frac{\left\|\boldsymbol{v}_{\text {oo }}^{\alpha \beta \gamma}\right\|}{\cos \theta_{\text {ref }}}$.

(iii) Saturate the reference by $\boldsymbol{u}_{\mathrm{f}, \text { ref }}^{\alpha \beta \gamma}=\left\|\boldsymbol{u}_{\mathrm{f}}^{\alpha \beta \gamma}\right\|_{\max } \cdot \frac{\boldsymbol{u}_{\mathrm{f}, \mathrm{ref}}^{\alpha \beta \gamma}}{\left\|\boldsymbol{u}_{\mathrm{f}, \text { ref }}^{\alpha \beta \gamma}\right\|}$.

This 3D-SVM leads to a possible increase of the synthesized vector amplitude up to $\frac{2}{3} u_{\mathrm{g}}^{\mathrm{dc}}$ when projected onto the $(\alpha, \beta)$ plane compared to the maximal amplitude $\frac{1}{2} u_{\mathrm{g}}^{\mathrm{dc}}$ for PWM.

\section{State-feedback current and voltage controller}

A discrete-time PI-SFC based on LQR theory with decoupling compensation similar to [12] is designed. It is assumed that the reference frame is ideally synchronized with the grid and that its frequency is constant. Controllability and observability are satisfied for positive LC filter parameters.

1) Filter current decoupling control: As introduced in [12], the cross-coupling and the inter-coupling of the controlled current shall be decoupled by introducing the filter current decoupling controller (FCDC), i.e.

$$
\boldsymbol{u}_{\mathrm{FCDC}}=\underbrace{\beta\left[\begin{array}{cccccc}
0 & -\omega_{\mathrm{g}} & 0 & \frac{1}{L_{\mathrm{f}}} & 0 & 0 \\
\omega_{\mathrm{g}} & 0 & 0 & 0 & \frac{1}{L_{\mathrm{f}}} & 0 \\
0 & 0 & 0 & 0 & 0 & 0
\end{array}\right]}_{:=\boldsymbol{A}_{\mathbf{F C D C}}} L_{\mathrm{f}} \boldsymbol{x},
$$

where $\beta=1$ is chosen in this work in order to achieve a full decoupling. The introduction of the decoupling based on system states in fact modifies the system matrix in (9) to

$$
\boldsymbol{A}_{\mathrm{m}}=\boldsymbol{A}+\boldsymbol{A}_{\mathrm{FCDC}} \text {. }
$$

2) Discretization: To adapt to the discrete-time nature of practical controllers, the system is discretized with sampling time $T_{\mathrm{S}}$ using exact discretization yielding

$$
\begin{aligned}
\boldsymbol{x}[k+1] & =\boldsymbol{A}_{\mathrm{k}} \boldsymbol{x}[k]+\boldsymbol{B}_{\mathrm{k}} \boldsymbol{u}[k]+\boldsymbol{B}_{\mathrm{dk}} \boldsymbol{u}_{\mathrm{d}}[k], \boldsymbol{x}[0]=\boldsymbol{x}_{0}, \\
\boldsymbol{y}[k] & =\boldsymbol{C}_{\mathrm{k}} \boldsymbol{x}[k],
\end{aligned}
$$

with $k$ denoting the discrete-time index $\boldsymbol{x}[k]:=\boldsymbol{x}\left(k T_{\mathrm{S}}\right)$. The discrete-time system matrices are given by

$$
\begin{aligned}
& \boldsymbol{A}_{\mathrm{k}}=e^{\boldsymbol{A}_{\mathrm{m}} T_{\mathrm{s}}}, \quad \boldsymbol{B}_{\mathrm{k}}=\boldsymbol{A}_{\mathrm{m}}^{-1}\left(\boldsymbol{A}_{\mathrm{k}}-\boldsymbol{I}_{6}\right) \boldsymbol{B} \\
& \boldsymbol{C}_{\mathrm{k}}=\boldsymbol{C} \quad \text { and } \quad \boldsymbol{B}_{\mathrm{dk}}=\boldsymbol{A}_{\mathrm{m}}^{-1}\left(\boldsymbol{A}_{\mathrm{k}}-\boldsymbol{I}_{6}\right) \boldsymbol{B}_{\mathrm{d}}
\end{aligned}
$$

3) Integral control action: An integral action is added to achieve zero steady-state error. Thus, the system has to be extended by extra integral states $\boldsymbol{\xi}:=\left(\xi_{i_{\mathrm{f}}^{d}}, \xi_{i_{\mathrm{f}}^{q}}, \xi_{u_{\mathrm{f}}^{\gamma}}\right)^{\top} \in \mathbb{R}^{3}$, i.e.

$$
\boldsymbol{\xi}[k+1]=\boldsymbol{\xi}[k]+T_{\mathrm{s}}\left(\boldsymbol{y}_{\mathrm{ref}}[k]-\boldsymbol{C}_{\mathrm{k}} \boldsymbol{x}[k]\right),
$$

which, with (16), gives the extended system

$$
\begin{aligned}
\underbrace{\left(\begin{array}{c}
\boldsymbol{x}[k+1] \\
\boldsymbol{\xi}[k+1]
\end{array}\right)}_{\boldsymbol{x}_{\xi}[k+1]} & =\underbrace{\left[\begin{array}{cc}
\boldsymbol{A}_{\mathrm{k}} & \boldsymbol{O}_{6 \times 3} \\
-T_{\mathrm{s}} \boldsymbol{C}_{\mathrm{k}} & \boldsymbol{I}_{3}
\end{array}\right]}_{:=\boldsymbol{A}_{\xi}}\left(\begin{array}{c}
\boldsymbol{x}[k] \\
\boldsymbol{\xi}[k]
\end{array}\right)+\underbrace{\left[\begin{array}{c}
\boldsymbol{B}_{\mathrm{k}} \\
\boldsymbol{O}_{3 \times 3}
\end{array}\right]}_{:=\boldsymbol{B}_{\xi}} \boldsymbol{u}[k] \\
& +\underbrace{\left[\begin{array}{c}
\boldsymbol{B}_{\mathrm{dk}} \\
\boldsymbol{O}_{3 \times 3}
\end{array}\right]}_{:=\boldsymbol{B}_{\mathrm{d} \xi}} \boldsymbol{u}_{\mathrm{d}}[k]+\underbrace{\left[\begin{array}{c}
\boldsymbol{O}_{6 \times 3} \\
T_{\mathrm{s}} \boldsymbol{I}_{3}
\end{array}\right]}_{:=\boldsymbol{E}_{\xi}} \boldsymbol{y}_{\mathrm{ref}}[k], \\
\boldsymbol{y}[k] & =\underbrace{\left[\begin{array}{ll}
\boldsymbol{C}_{\mathrm{c}} & \boldsymbol{O}_{3 \times 3}
\end{array}\right]}_{:=\boldsymbol{C}_{\boldsymbol{\xi}}} \boldsymbol{x}_{\xi}[k],
\end{aligned}
$$

with the augmented state vector $\boldsymbol{x}_{\xi}:=\left(\boldsymbol{x}^{\top}, \boldsymbol{\xi}^{\top}\right)^{\top} \in \mathbb{R}^{9}$.

4) PI state-feedback controller with anti-windup: By defining the control law

$$
\boldsymbol{u}_{\text {ref }}[k]=-\underbrace{\left[\begin{array}{ll}
\boldsymbol{K}_{\mathrm{x}} & \boldsymbol{K}_{\mathrm{i}}
\end{array}\right]}_{\boldsymbol{K}} \boldsymbol{x}_{\xi}[k]+\boldsymbol{K}_{\mathrm{p}}\left(\boldsymbol{y}_{\mathrm{ref}}[k]-\boldsymbol{C}_{\xi} \boldsymbol{x}_{\xi}[k]\right),
$$

the controller exhibits a PI-like behavior. The gain matrix $\boldsymbol{K}$ is determined based on LQR theory for the cost function

$$
J=\frac{1}{2} \sum_{k=0}^{N}\left(\boldsymbol{x}_{\xi}^{\top}[k] \boldsymbol{Q} \boldsymbol{x}_{\xi}[k]+\boldsymbol{u}_{\mathrm{ref}}^{\top}[k] \boldsymbol{R} \boldsymbol{u}_{\mathrm{ref}}[k]\right),
$$

where $\boldsymbol{Q}$ and $\boldsymbol{R}$ denote the weighting matrices of states and input, which are parameterized according to Bryson's Rule for the initial iteration [18]. $\boldsymbol{K}_{\mathrm{p}}$ is designed similarly to [12], but for the discrete system, as follows

$$
\boldsymbol{K}_{\mathrm{p}}=-\left(\boldsymbol{C}_{\mathrm{k}}\left(\boldsymbol{A}_{\mathrm{k}}-\boldsymbol{I}_{6}\right)^{-1} \boldsymbol{B}_{\mathrm{k}}\right)^{-1} .
$$


TABLE I: Parameters of system and implementation.

\begin{tabular}{lcc}
\hline Name & Parameter & Value \\
\hline DC-link voltage & $u_{\mathrm{g}}^{\mathrm{dc}}$ & $700 \mathrm{~V}$ \\
DC-link capacitance & $C_{\mathrm{g}}$ & $810 \mu \mathrm{F}$ \\
Balancing Resistors & $R_{\mathrm{b}}$ & $250 \mathrm{k} \Omega$ \\
Switching frequency & $f_{\mathrm{sw}}$ & $70 \mathrm{kHz}$ \\
Filter resistance & $R_{\mathrm{f}}$ & $220 \mathrm{~m} \Omega$ \\
Filter inductance & $L_{\mathrm{f}}$ & $820 \mu \mathrm{H}$ \\
Equivalent series resistance & $R_{\mathrm{c}}$ & $0 \Omega$ \\
Filter capacitance & $C_{\mathrm{f}}$ & $4.7 \mu \mathrm{F}$ \\
Grid resistance & $R_{\mathrm{g}}$ & $0 \Omega$ \\
Grid inductance & $L_{\mathrm{g}}$ & $200 \mu \mathrm{H}$ \\
Grid frequency & $\omega_{\mathrm{g}}$ & $2 \pi \cdot 50 \mathrm{rad} / \mathrm{s}$ \\
Grid nominal voltage & $\hat{u}_{\mathrm{g}}$ & $\sqrt{2} \cdot 230 \mathrm{~V}$ \\
\hline State weighting & $\boldsymbol{Q}$ & $\operatorname{diag}\left(0.18 \boldsymbol{I}_{3}, 8.16 \mathrm{e}-5 \boldsymbol{I}_{3}\right.$, \\
& & $\left.1 \mathrm{e} 8 \boldsymbol{I}_{2}, 4.6 \mathrm{e} 5\right)$ \\
Input weighting & $\boldsymbol{R}$ & $8.16 \mathrm{e}-5 \boldsymbol{I}_{3}$ \\
\hline Simulation rate & - & $7 \mathrm{MHz}$ \\
Simulink solver & - & ode 4 \\
\hline
\end{tabular}

In order to alleviate overshooting and oscillations, an antiwindup strategy is applied based on the dynamic saturation detected in modulation: the error integrator stops once the reference amplitude exceeds the modulation cube [17].

5) Delay compensation: The reference output voltage will be applied by the VSI with one time step delay, i.e. $\boldsymbol{u}_{\mathrm{f}}^{\alpha \beta \gamma}[k]=$ $\boldsymbol{u}_{\mathrm{f}, \mathrm{ref}}^{d q \gamma}[k-1]$ if the nonlinear effect in the VSI is not considered. This delay can be compensated for during Park transformation by adding the angle $\omega_{\mathrm{g}} T_{\mathrm{s}}$ to the actual transformation angle to rotate the reference vector one step forward, which gives

$$
\boldsymbol{u}_{\mathrm{f}, \mathrm{ref}}^{\alpha \beta \gamma}[k]=\boldsymbol{T}_{\mathrm{p}}\left(\phi_{\mathrm{g}}[k]+\omega_{\mathrm{g}} T_{\mathrm{s}}\right) \boldsymbol{u}_{\mathrm{ref}}[k] .
$$

\section{IMPLEMENTATION AND RESULTS}

The designed PI state-feedback controller and the 3DSVM for the proposed topology are implemented in MATLAB/Simulink and were verified in PLECS. The system is implemented according to Section II with the parameters given in Tab. I. For comparison, a conventional PI controller for $\boldsymbol{i}_{\mathrm{f}}^{d q}$, designed based on magnitude optimum, is implemented for the same system, without controlling $u_{\mathrm{c}}^{\gamma}$. A feed-forward disturbance compensation and decoupling is also implemented [19]. The simulation results are shown in Fig. 3. They are divided into four intervals, where each one takes $80 \mathrm{~ms}$ with different grid conditions. The current reference is chosen so that each condition appears and only one step occurs at a time and repeats in each interval. With ideal synchronization, the grid condition starts with symmetrical and sinusoidal voltage. Subsequently, an one-phase failure is simulated. In the third interval, the 5 th harmonic is added having amplitude of $5 \%$ of the nominal value. The worst case is shown in the last interval, where the failed phase comes back but the harmonic distortion remains. There, the VSI needs to apply the longest voltage vectors and thus reaches its voltage limits during modulation.

For both controllers, $\boldsymbol{i}_{\mathrm{f}}^{d q}$ is nicely controlled under each grid condition. However, a difference can be noticed during current reference steps. The LQR controller shows a faster control performance, especially for the $d$-component, which happens for example at $45 \mathrm{~ms}$ and $125 \mathrm{~ms}$ where $i_{\mathrm{f}}^{d}$ steps from $-20 \mathrm{~A}$ to $+20 \mathrm{~A}$. The advantage is also demonstrated in the decoupling capability, visible at $45 \mathrm{~ms}$ and $185 \mathrm{~ms}$, where $i_{\mathrm{f}}^{q}$ experiences smaller errors for the PI-SFC than for the PI controller. Furthermore, facing abrupt grid distortions at $80 \mathrm{~ms}$ and $240 \mathrm{~ms}$, where phase a was lost and brought back, the LQR shows better damping characteristics. In addition to the shorter settling time, the implementation of 3D-SVM has also contributed to faster dynamics due to the enlarged feasible modulation domain (full exploitation of the cube). As shown in the last subplot, while the maximal reference amplitude in $(\alpha, \beta)$-plane remains the same with $\frac{1}{2} u_{\mathrm{g}}^{\mathrm{dc}}$ for PWM, the amplitude limit of the 3D-SVM varies, depending on the orientation of the reference voltage vector and allows for larger reference amplitudes than the PWM limit. This indicates more margin available for large reference steps, better decoupling, and disturbance rejection.

Besides the $\boldsymbol{i}_{\mathrm{f}}^{d q}$ control performance, the voltage $u_{\mathrm{c}}^{\gamma}$ is significantly different for both controllers. As, $u_{\mathrm{f}}^{\gamma}$ is set to zero by the PI controller, the uncontrolled $u_{\mathrm{c}}^{\gamma}$ shows pronounced oscillations with an amplitude of up to $\pm 20 \mathrm{~V}$ due to modulation. In comparison, the LQR controller can keep $u_{\mathrm{c}}^{\gamma}$ close to zero, and thus, provides a better performance and lower losses by minimizing $\left\|i_{\mathrm{f}}^{\gamma}\right\|$ as in (11).

\section{CONCLUSion}

A novel three-phase grid-side voltage source inverter topology with split DC-link capacitors is introduced and analysed. The main difference to the known topologies is, that the midpoint is not connected to the neutral point of the grid. However, the LC-filter star-point is connected to the midpoint of the split DC-link, which allows for a $\gamma$-component to flow through the filter and into the DC-link. To reduce losses and minimize fluctuations, this $\gamma$-component is controlled to zero. A detailed state-space model is derived, which is used for state-feedback controller design. As modulation technique, a 3D-SVM with dynamic saturation is proposed which enables the full utilisation of modulation domain and thus implies significantly improved control, decoupling and disturbance rejection performance. Model and state-feedback controller are implemented and the results are compared to a standard PI controller. The state-feedback controller shows a significantly better and faster dynamic response to reference steps, as well as less cross coupling. Most important, the $\gamma$-component could be kept close to zero with this approach.

\section{REFERENCES}

[1] C. Hackl, C. Dirscherl, and K. Schechner, "Modellierung und Regelung von modernen Windkraftanlagen," in Elektrische Antriebe - Regelung von Antriebssystemen, D. Schröder, Ed. Springer-Verlag, 2021, ch. 10.3 , pp. 1504-1585.

[2] H. Abu-Rub, J. Holtz, J. Rodriguez, and G. Baoming, "Medium-Voltage Multilevel Converters-State of the Art, Challenges, and Requirements in Industrial Applications," IEEE Transactions on Industrial Electronics, vol. 57, no. 8, pp. 2581-2596, Aug 2010. 


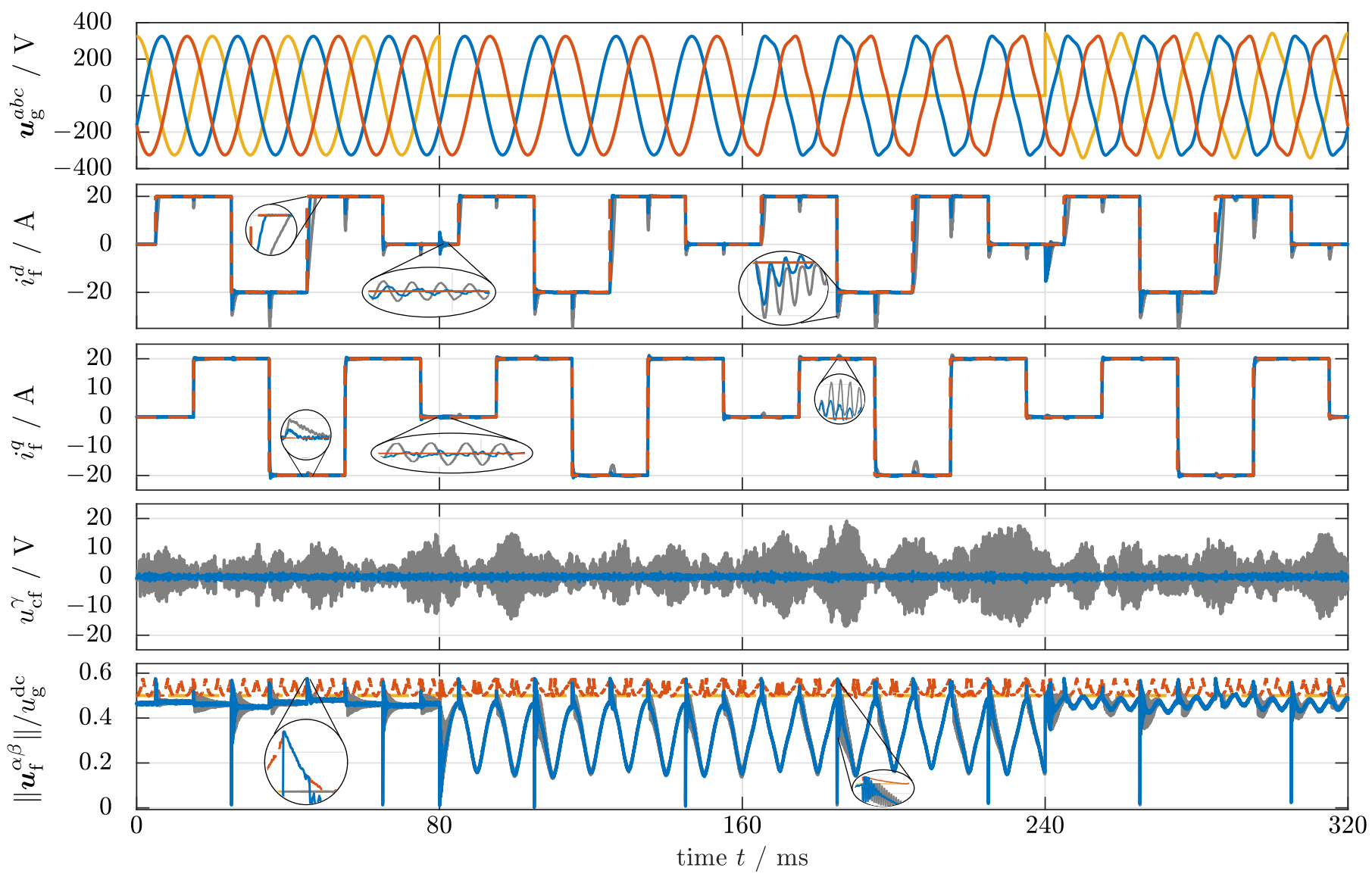

Fig. 3: Comparison of the simulation results, where grid voltages $u_{\mathrm{g}}^{a}[-], u_{\mathrm{g}}^{b}[-]$ and $u_{\mathrm{g}}^{c}[-]$ are at the top. In the other subplots, the performances of the LQR controller with 3D-SVM [-] and PI controller with PWM [-] are shown. In the subplots where the currents $i_{\mathrm{f}}^{d q}$ are shown, the red dashed line [---] is the reference. The last subplot shows the voltage amplitudes where the voltage limits are represented by the yellow $\left[-{ }^{--}\right]$and red $[-=]$dashed lines for 3D-SVM and PWM, respectively.

[3] C. Wang and Y. Li, "Analysis and Calculation of Zero-Sequence Voltage Considering Neutral-Point Potential Balancing in Three-Level NPC Converters," IEEE Transactions on Industrial Electronics, vol. 57, no. 7 , pp. 2262-2271, 2010.

[4] L. Hassaine, E. OLias, J. Quintero, and V. Salas, "Overview of power inverter topologies and control structures for grid connected photovoltaic systems," Renewable and Sustainable Energy Reviews, vol. 30, pp. 796 $-807,2014$.

[5] R. Teodorescu, M. Liserre, and P. Rodríguez, Grid Converters for Photovoltaic and Wind Power Systems, U. K. Chichester, Ed. John Wiley \& Sons, Ltd, 2011.

[6] M. Liserre, F. Blaabjerg, and S. Hansen, "Design and control of an LCLfilter-based three-phase active rectifier," IEEE Transactions on Industry Applications, vol. 41, no. 5, pp. 1281-1291, 2005.

[7] M. Liserre, R. Teodorescu, and F. Blaabjerg, "Stability of Photovoltaic and Wind Turbine Grid-Connected Inverters for a Large Set of Grid Impedance Values," IEEE Transactions on Power Electronics, vol. 21, no. 1 , pp. 263-272, 2006.

[8] N. P. W. Strachan and D. Jovcic, "Stability of a Variable-Speed Permanent Magnet Wind Generator With Weak AC Grids," IEEE Transactions on Power Delivery, vol. 25, no. 4, pp. 2779-2788, 2010.

[9] R. Luhtala, T. Roinila, and T. Messo, "Implementation of Real-Time Impedance-Based Stability Assessment of Grid-Connected Systems Using MIMO-Identification Techniques," IEEE Transactions on Industry Applications, vol. 54, no. 5, pp. 5054-5063, 2018.

[10] S. Grunau and F. W. Fuchs, "Effect of Wind-Energy Power Injection into Weak Grids," European Wind Energy Conference and Exhibition 2012, EWEC 2012, vol. 2, pp. 1150-1156, 012012.

[11] J. Kukkola, M. Hinkkanen, and K. Zenger, "Observer-Based State-Space

Current Controller for a Grid Converter Equipped With an LCL Filter: Analytical Method for Direct Discrete-Time Design," IEEE Transactions on Industry Applications, vol. 51, no. 5, pp. 4079-4090, 2015.

[12] C. Dirscherl, J. Fessler, C. M. Hackl, and H. Ipach, "State-feedback controller and observer design for grid-connected voltage source power converters with LCL-filter," in 2015 IEEE Conference on Control Applications (CCA), Sep. 2015, pp. 215-222.

[13] M. G. Villalva and F. Ruppert, "3-D Space Vector PWM for Three-Leg Four-Wire Voltage Source Inverters," in 2004 IEEE 35th Annual Power Electronics Specialists Conference (IEEE Cat. No.04CH37551), vol. 5, June 2004, pp. 3946-3951 Vol.5.

[14] S. Krüner and C. M. Hackl, "Experimental Identification of the Optimal Current Vectors for a Permanent-Magnet Synchronous Machine in Wave Energy Converters," Energies, vol. 12, no. 5, 2019.

[15] J. Dannehl, M. Liserre, and F. W. Fuchs, "Filter-Based Active Damping of Voltage Source Converters With LCL Filter," IEEE Transactions on Industrial Electronics, vol. 58, no. 8, pp. 3623-3633, 2011.

[16] C. M. Hackl and M. Landerer, "A unified method for generic signal parameter estimation of arbitrarily distorted single-phase grids with dcoffset," IEEE Open Journal of the Industrial Electronics Society, vol. 1, pp. 235-246, 2020.

[17] C. M. Hackl, U. Pecha, and K. Schechner, "Modeling and control of permanent-magnet synchronous generators under open-switch converter faults," IEEE Transactions on Power Electronics, 2018.

[18] A. Bryson, Applied optimal control : optimization, estimation, and control. New York Oxon: CRC Press LLC Routledge, 2018

[19] C. M. Hackl, Non-identifier based adaptive control in mechatronics: Theory and Application, ser. Lecture Notes in Control and Information Sciences. Springer International Publishing, 2017, no. 466. 